\title{
Índices de estresse do trabalhador de saúde mental
}

\author{
Mental health worker stress indexes \\ Índices de estrés del trabajador de salud mental \\ Pedro Martini Bonaldo ${ }^{1}$, Carlos Eduardo Portela ${ }^{1 *}$.
}

\begin{abstract}
RESUMO
Objetivo: Esta pesquisa tem por objetivo mensurar o nível de estresse do trabalhador de saúde mental. Sendo seus objetivos específicos: Interpretar e avaliar os indicadores de saúde mental e depressão dos trabalhadores de saúde mental e articular os dados presentes nos três instrumentos para uma maior compreensão do sujeito. Métodos: A pesquisa aconteceu em um hospital psiquiátrico do Distrito Federal e envolveu 10 profissionais da saúde. Os instrumentos aplicados foram os Inventário de sintomas de stress para adultos de Lipp (ISSL), Escala de depressão de Beck (BDI) e Questionário de Saúde Geral de Goldberg (QSG). Resultados: Os resultados mostram que 70\% desta amostra já se encontrava em uma fase avançada do estresse, com uma sintomatologia física e psíquica. Os resultados mostram que dos dez sujeitos de pesquisa, dois apresentaram depressão moderada e três apresentaram depressão leve. Os outros cinco participantes embora tivessem sintomas depressivos não foi suficiente para caracterizar uma depressão leve ou outro quadro mais grave. Conclusão: Esses dados problematizam a necessidade de serem criadas estratégias para cuidar da saúde mental do profissional de saúde mental.
\end{abstract}

Palavras-chave: Estresse, Saúde mental, Profissionais de saúde.

\begin{abstract}
Objective: This research aims to measure the level of stress of the mental health worker. Its specific objectives are: Interpreting and evaluating the mental health and depression indicators of mental health workers and articulating the data present in the three instruments for a greater understanding of the subject Method: The research took place in a psychiatric hospital in the Federal District and involved 10 professionals of health. The instruments applied were the Lipp's Inventory of Stress Symptoms for Adults (ISSL), Beck's Depression Scale (BDI) and Goldberg's General Health Questionnaire (QSG). Results: The results show that $70 \%$ of this sample was already in an advanced stage of stress, with physical and psychological symptoms. The results show that of the ten research subjects, two had moderate depression and three had mild depression. The other five participants, although they had depressive symptoms, was not enough to characterize mild depression or another more severe condition. Conclusion: These data problematize the need to create strategies to care for the mental health of the mental health professional.
\end{abstract}

Key words: Stress, Mental health, Health professional.

\section{RESUMEN}

Objetivo: Esta investigación tiene como objetivo medir el nivel de estrés del trabajador de salud mental. Sus objetivos específicos: interpretar y evaluar los indicadores de salud mental y depresión de los trabajadores de salud mental y articular los datos presentes en los tres instrumentos para una mayor comprensión del tema Método: La investigación se llevó a cabo en un hospital psiquiátrico en el Distrito Federal e involucró a

1Escola Superior de Ciências da Saúde. Brasília - DF. `E-mail: carlosedupsi@gmail.com

SUBMETIDO EM: 04/2020

ACEITO EM: 5/2020

PUBLICADO EM: 7/2020

REAS/EJCH | Vol.Sup.n.52 | e3702 | DOI: https://doi.org/10.25248/reas.e3702.2020 Página 1 de 8 
10 profesionales salud Los instrumentos aplicados fueron el Inventario de síntomas de estrés para adultos de Lipp (ISSL), la Escala de depresión de Beck (BDI) y el Cuestionario general de salud de Goldberg (QSG). Resultados: Los resultados muestran que el $70 \%$ de esta muestra ya se encontraba en una etapa avanzada de estrés, con síntomas físicos y psicológicos. Los resultados muestran que de los diez sujetos de investigación, dos tenían depresión moderada y tres tenían depresión leve. Los otros cinco participantes, aunque tenían síntomas depresivos, no fueron suficientes para caracterizar la depresión leve u otra afección más grave. Conclusión: estos datos problematizan la necesidad de crear estrategias para cuidar la salud mental del profesional de la salud mental.

Palabras clave: Estrés, Salud mental, Profesionales de la salud.

\section{INTRODUÇÃO}

O conceito de saúde é antigo, porém com o passar do tempo ele mudou e se atualizou. De acordo com Straub RO (2003), as primeiras representações da medicina datam de vinte mil anos com pinturas em cavernas que mostram xamãs com máscaras e práticas de curandeiro. Conforme o domínio da medicina começa a progredir, durante a primeira metade do século XX, o fazer em saúdes e sustenta cada vez mais na anatomia e fisiologia, ao invés de estudar as emoções e pensamentos, que teria como consequência a compreensão da maior complexidade e integralidade da saúde. Esse modelo se mantém hegemônico até os dias de hoje. Com o passar dos anos passa-se a pensar saúde pelo modelo biopsicossocial que reconhece que forças biológicas, psicológicas e socioculturais agem em conjunto com a saúde e a vulnerabilidade do indivíduo à doença. Em outras palavras, a saúde e doença precisam ser explicadas por contextos múltiplos e imbricados (STRAUB RO, 2005).

Um dos conceitos mais importantes para se compreender a saúde pelo modelo biopsicossocial é o estresse e, ironicamente, não existe ainda na literatura uma definição unívoca do que é estresse. De acordo com Rossetti MO, et al. (2008), o estresse é uma reação do organismo que inclui os aspectos físicos, psíquicos e hormonais perante uma situação que requeira uma grande adaptação deste a um evento estressor, mas que será prejudicial caso tenha uma exposição prolongada ou uma predisposição do indivíduo para determinada doença.

O estresse tem características particulares que são descritas na literatura como tendo a capacidade de diminuir nossa imunidade ao mesmo tempo que um nível mínimo de estresse pode ser benéfico. De acordo com Straub RO (2003), o estresse tem algumas características importantes: a primeira é que o corpo tem uma resposta semelhante a diversos estressores diferentes; a segunda é que o estresse, às vezes, pode deixar as pessoas doentes. Este tópico é de grande relevância pois, embora o estresse não seja a doença em si, pesquisas mostram que pessoas expostas por um estresse crônico tendem a ficar com a vulnerabilidade rebaixada para o desenvolvimento de doenças como: câncer, cardiopatia, diabetes, artrites, cefaleias, asma, distúrbios digestivos, depressão e ansiedade. Por outro lado, um nível de estresse baixo pode ser positivo em nossas vidas e nos deixar com mais recursos de enfrentamento para o futuro.

O modelo de estresse proposto por Selye H (1951), baseado nas fases de alarme, resistência e exaustão inspirou Lipp MEN (2005), a criar o "Inventário de sintomas de stress para adultos de Lipp" (ISSL), ela propõe um modelo quadrifásico do estresse. O modelo de Lipp MEN (2003), tem grandes semelhanças com o de Selye H (1959), com a diferença da maior ênfase que a autora dá aos aspectos psicológicos do estresse (todavia mantém a concepção do estresse manifestado por sintomas físicos) e adiciona uma nova fase do estresse conhecida como quase-exaustão. De acordo com Lipp MEN e Lipp LMN (2019), a fase alerta se refere ao estresse positivo, o sujeito se energiza para enfrentar aquela situação. Podem ocorrer mudanças fisiológicas como a liberação da adrenalina, o dilatamento da pupila dentre outras reações, a sobrevivência é preservada e uma sensação de plenitude é frequentemente alcançada. A fase de resistência é quando sujeito tenta lidar com os estressores ao mesmo tempo que mantém sua homeostase interna, se os estressores persistirem em termos de frequência e intensidade, então existe uma quebra da resistência e o sujeito entra na fase de quase-exaustão. 
$\mathrm{Na}$ fase de quase-exaustão é onde o adoecimento se inicia e a vulnerabilidade genética ou adquirida pode aparecer, seja por meio de aparecimento de síndromes que se aproveitam da baixa vulnerabilidade, seja por órgãos mais sensíveis se manifestarem. Se não há alívio em relação ao estresse por meio do afastamento dos estressores ou pelo uso das estratégias de enfrentamento, então o sujeito chega na última fase de estresse, a exaustão. A fase de exaustão é quando doenças graves atingem órgãos mais vulneráveis como úlceras, enfartes, psoríase, e outras, além de manifestações psicológicas como depressão, ansiedade, etc. (LIPP MEN, 2005).

Em termos epidemiológicos o estresse já atinge boa parte da população, se transformando em um problema de saúde pública. A pesquisa de Lipp MEN (2012), feita com mais de 2000 brasileiros com idade de 18 à 75 anos que responderam a uma escala que mensurava o nível de estresse encontrou os seguintes dados: $34,26 \%$ dos entrevistados estavam com o estresse no nível máximo ( notas 8-10, numa escala de 10 pontos); 4,02\% dos participantes atingiram níveis de estresse extremos (10 pontos); Os brasileiros utilizam estratégias de enfrentamento desde conversar com a família e amigos sobre o problema até procurar um psicólogo, acupunturista, rezar, fazer compras e comer, tentando manter seu nível de estresse dentro do que eles acreditam ser o normal (níveis 5-6 na escala de 10 pontos); Mais de um terço dos participantes disse que o estresse aumentou ultimamente; Os relacionamentos (familiares, amorosos, com colegas e chefes) são apontados como os maiores estressores; sobrecarga no trabalho é o terceiro maior estressor; $52,28 \%$ tiveram ou têm o diagnóstico de estresse; $55,60 \%$ sofrem de ansiedade, $23,20 \%$ sofrem ou sofreram de depressão e 10,37\% têm ou já tiveram pânico.

O trabalho em saúde, sobretudo em hospitais exige por parte dos trabalhadores desafios para manejo do estresse ocupacional como: ambiente insalubres, regime de trabalho divido em turnos, salários baixos e contato próximo com pessoas em adoecimento físico e psíquico. Em um trabalho divulgado por Dias S, et al. (2010), foi encontrado na sua amostra de profissionais de saúde índices alarmantes de adoecimento: $59,8 \%$ apresentam baixos níveis de realização profissional, 2,7\% apresentavam exaustão emocional e 0,9\% o sintoma de despersonalização.

Em estudo realizado por Camelo SH e Angerami ELS (2004), com profissionais da saúde em uma equipe multidisciplinar no programa saúde da família encontrou os seguintes índices: $62 \%$ dos trabalhadores apresentavam-se com estresse, sendo deles $83 \%$ na fase resistência e $17 \%$ na fase quaseexaustão. Mais uma vez esses índices são bastantes preocupantes, visto que a fase resistência já é constituída por uma manifestação sintomática física e psíquica do estresse.

Segundo Fernandes MA e Marziale MHP (2014) 2,34 milhões de pessoas morrem todos os anos por acidentes no trabalho e doenças relacionadas ao trabalho. Dessas 2,02 milhões (86,3\%) são causados por doenças profissionais (profissões que fazem com que as pessoas permaneçam em contato contínuo com fatores de risco para o adoecimento sejam, físicos, químicos e psíquicos) e 321 de acidentes de trabalho. São 6300 mortes diárias relacionadas ao trabalho, 5500 causadas por doenças profissionais; números muito elevados. Estes números indicam que ações devem existir para que tais condições sejam reparadas e se consiga condições dignas do trabalho (remuneração adequada, possibilidade de autonomia e liberdade no trabalho, equidade e segurança, além de que o trabalho garanta uma condição de uma vida digna às pessoas).

Diante destes dados preocupantes da população, vê-se a grande relevância de se estudar o estresse até para se encontrar estratégias de enfrentamento para diminuir o nível de estresse da população para assim evitar o seu adoecimento. Além disto, entende-se que estudos como estes podem embasar políticas públicas de saúde de prevenção, promoção e remediação. No caso dos trabalhadores de saúde como citado por Camelo SH e Angerami ELS (2004), a hipótese é que seu nível de estresse está maior por ele ser muitas vezes exposto a situações de adoecimento psíquico grave que requer estratégias de enfrentamento assertivas. Sendo assim o presente estudo tem como objetivo principal mensurar o nível de estresse do trabalhador de saúde mental. Os objetivos específicos são: Interpretar e avaliar os indicadores de saúde mental e depressão dos trabalhadores de saúde mental e articular os dados presentes nos três instrumentos da pesquisa para uma maior compreensão do sujeito. Evidentemente a saúde tem diversos

REAS/EJCH | Vol.Sup.n.52 | e3702 | DOI: https://doi.org/10.25248/reas.e3702.2020 Página 3 de 8 
determinantes e o estresse é um componente importante para a saúde do trabalhador. Além disto, este trabalho propõe pensar saúde em um modelo ampliado e integral. Pensar em uma saúde integral e em um modelo ampliado de saúde é pensar na saúde como tendo diversos condicionantes e determinantes como por exemplos: saneamento básico, educação, meio ambiente, o trabalho, o lazer, dentre outros (BRASIL, 1990).

\section{MÉTODOS}

A pesquisa se aproxima mais de uma metodologia quantitativa, visto que tem como objetivo falar sobre diferentes índices de uma referida população. Para isto, aplicou-se instrumentos validados no Brasil para mensurar fatores como estresse, depressão e falar da saúde mental de maneira global. Este trabalho foi aprovado pelo Comitê de Ética em Pesquisa da Fundação de Ensino e Pesquisa em Ciências da Saúde (FEPECS) com parecer ํㅜ : 1.932.683.

A pesquisa foi realizada em um hospital psiquiátrico do Distrito Federal. Os participantes da pesquisa são profissionais de saúde que trabalham em um hospital Psiquiátrico. No caso do estudo, foram 10 profissionais da saúde, sendo 6 mulheres e 4 homens. Sendo 6 técnicos de enfermagem (quatro mulheres e dois homens) e 4 (duas mulheres e dois homens) enfermeiros. Dos 10 participantes, 6 tinham uma jornada de trabalho de 40 horas e 4 de 20 horas. Os instrumentos aplicados foram os Inventário de sintomas de stress para adultos de Lipp (ISSL), Escala de depressão de Beck (BDI) e Questionário de Saúde Geral de Goldberg (QSG). A escolha destes instrumentos se deu pela familiaridade do autor com o instrumento, para atingir os objetivos da pesquisa e por serem instrumentos validados e de grande relevância cientifica no país.

O Inventário de sintomas de stress para adulto de Lipp (ISSL) de acordo com Rossetti MO, et al. (2008), fornece uma medida objetiva dos sintomas de estresse em jovens acima de 15 anos e adultos. $O$ instrumento é formado por três eixos de avaliação frente às fases do estresse. O primeiro eixo contém 15 itens e se referem aos sintomas do estresse físicos e psicológico que a pessoa experimentou nas últimas 24 horas. O segundo, composto por dez sintomas físicos e cinco psíquicos que a pessoa experimentou na última semana. O terceiro eixo, é composto por 12 sintomas físicos e 11 psicológicos em que o sujeito experimentou no último mês. No total o teste apresenta 37 itens de natureza somática e 19 psicológicas, sendo em alguns momentos os sintomas se repetem na escala, diferindo na sua intensidade e seriedade.

O Inventário de depressão desenvolvido por Beck AT, et al. (1961). De acordo com Ely P, et al. (2014), o inventário foi desenvolvido para avaliar os sintomas de depressão, sendo usado para avaliar a intensidade destes sintomas depressivos, tanto para clínica quanto para pesquisa. Os itens presentes na escala se referem a insatisfação, punição, sentimento de fracasso, tristeza, pessimismo, auto aversão, retraimento social, insônia, pessimismo, ideias suicidas, indecisão, mudança na autoimagem, choro, dificuldade de trabalhar, perda de apetite e peso, perda de libido, fatigabilidade e preocupações somáticas. O inventário pode ser usado com pessoas de 17 a 80 anos. O teste é composto por 21 itens e com o objetivo de medir a intensidade da depressão. A pontuação da escala é do tipo Likert de 4 pontos (0, 1, 2 e 3), podendo ter pontuações de 0 a 63, nos 21 itens.

O questionário de Saúde Geral de Goldberg de acordo com Pasquali L, et al. (1994), não é um questionário de saúde geral é, antes, um questionário apropriado para avaliar a saúde mental ou o bemestar psicológico, tendo fácil aplicação e compreensão dos dados. A escala é multidimensional e contém 60 itens sobre sintomas não psicóticos, englobados em 6 escalas que são: Stress Psíquico, desejo de morte, desconfiança no próprio desempenho, distúrbios de sono, distúrbios psicossomáticos e saúde geral.

O questionário apresenta perguntas com quatro alternativas e cada alternativa fornece uma pontuação de 1 a 4 do tipo escala Likert. Os pontos variam para cada escala, mas, geralmente quando uma escala apresenta uma média maior ou igual a 3 então indica a presença de um distúrbio. $O$ instrumento pode ser aplicado coletivamente ou individualmente tendo sido amplamente usado em pesquisas no Brasil. Não apresenta restrição de idade e nem limite de tempo. 
O procedimento da pesquisa deu se da seguinte forma: pesquisador solicitou autorização da direção da instituição, bem como submeteu o presente trabalho ao comitê de Ética da ESCS (Escola Superior de Ciências da Saúde) e tendo sido aprovado executou a pesquisa. A pesquisa se baseou em pedir para os profissionais responderem três instrumentos para avaliar o seu nível de estresse, depressão e saúde geral e saúde mental. Importante ressaltar que aos participantes foi oferecido o termo de consentimento livre e esclarecido bem como explicado os objetivos da pesquisa. Os participantes puderam desistir a qualquer momento da pesquisa. A pesquisa consistiu a princípio na revisão de literatura, utilizando-se do método da leitura, interpretação e associação entre os textos. Para isto consistiu em pesquisar na literatura científica, livros, artigos que tenham como tema estresse e saúde mental dos últimos anos. Ao serem levantados estes dados foi feito uma análise e existiu uma tentativa de compreender às perguntas previamente estabelecidas nos objetivos da pesquisa. Após a revisão de literatura foi feito uma discussão com o orientador, seguido da coleta de dados no cenário. Foi feita a análise de dados prosseguindo para a fase finalização e por último a revisão e apresentação deste artigo.

\section{RESULTADOS E DISCUSSÃO}

Os dados referentes a pesquisa de cada instrumento serão apresentados separadamente para depois serem unidos para uma compreensão mais integral do fenômeno. Para estudar a relação da frequência de profissionais da saúde que apresentavam sintomas de estresse, foram utilizadas frequência e porcentagem, e estes dados podem ser observados na Tabela 1.

Tabela 1 - Frequência de profissionais da saúde que apresentaram sintomas de estresse.

\begin{tabular}{ccc}
\hline & Frequência & Porcentagem \\
\hline Alerta & 0 & 0 \\
Resistência & 7 & 0,70 \\
Quase-exaustão & 0 & 0 \\
Exaustão & 0 & 0 \\
Sem estresse & 3 & 0,30 \\
Total & 10 & 1 \\
\hline
\end{tabular}

Fonte: Bonaldo PM e Portela CE, 2020.

Verificou-se que $70 \%$ (7) dos participantes estavam na fase do estresse conhecida como resistência, uma fase que já é considerada por Lipp MEN (2005) um tipo de estresse prejudicial com sintomas físicos e psíquicos presentes. Enquanto 30\% (3) deles não tinham estresse algum, não foi encontrado sujeitos que estivessem na fase de quase-exaustão ou exaustão. Importante ressaltar que a predominância na fase da resistência indica que a pessoa automaticamente está lidando com os estressores de modo a manter a homeostase interna (LIPP MEN, 2001). Em outro estudo com policiais federais de São Paulo, a fase da resistência foi também preponderante, mostrando como os profissionais da saúde podem estar lidando com estressores que embora qualitativamente diferentes, estão fazendo com que a equipe trabalhe com uma carga estressora tão alta quanto policiais federais (ROSSETTI MO, et al., 2008).

Um dado bastante interessante é que os três profissionais que não tinham estresse, todos eles eram profissionais que estavam em uma jornada de 20 horas semanais. Mostrando que os profissionais que tinham uma jornada de 40 horas no hospital, 6 deles estavam em uma fase do estresse conhecida como resistência e um deles que também estava nesta fase e tinha jornada de 20 horas. Mais uma vez vemos que os profissionais de saúde que trabalham no hospital pesquisado em uma jornada de trabalho 40 horas estão mais estressados, podemos pensar como a instituição contribui para níveis maiores de estresse desses trabalhadores. Importante também qualificar o tipo de manifestação desse estresse, se preponderou mais uma manifestação do estresse por um sintoma físico ou psíquico. Para estudar a relação da frequência de profissionais da saúde que apresentavam sintomas de estresse e o tipo da manifestação deste estresse (físico ou psíquico), foram utilizadas frequência e porcentagem, e estes dados podem ser observados na Tabela 2. 
Tabela 2 - Frequência de profissionais da saúde que apresentaram sintomas de estresse e suas manifestações (sintomas físicos ou psíquicos).

\begin{tabular}{ccc}
\hline & Frequência & Porcentagem \\
\hline Físico & 3 & 0,43 \\
Psíquico & 4 & 0,57 \\
Total & 7 & 1 \\
\hline
\end{tabular}

Fonte: Bonaldo PM e Portela CE, 2020.

Verificou-se que a manifestação psíquica (57\%) teve uma preponderância sobre a manifestação física (43\%), as manifestações psíquicas do estresse incluem irritabilidade, sensível, diminuição da libido, tristeza, dentre outras. Isto mostra uma área privilegiada para atuação com estes profissionais, sobretudo por meio da psicoterapia e programas que pudessem mitigar essas manifestações sintomáticas psíquicas.

Os participantes com ou sem estresse apresentaram manifestações físicas e psíquicas. Em termo de frequência observou-se que a tensão muscular e a insônia foram os sintomas físicos mais preponderantes nos participantes, 7 dos 10 participantes relataram tensão muscular ao final do dia e 6 dos 10 referiram insônia.

Os sintomas psíquicos do estresse também foram descritos e a irritabilidade excessiva apareceu com uma frequência de 6 vezes e a sensibilidade emotiva excessiva apareceu em 5 casos. Esses dados são bastante alarmantes porque mesmo os sujeitos julgados não tendo estresse pela escala ISSL, de alguma forma tiveram a presença de manifestações físicas e psíquicas do estresse, o que mais uma vez indica um sofrimento psíquico presente nestes trabalhadores.

A pesquisa também utilizou o inventário de depressão de Beck (BDI) para mensurar a intensidade e frequência dos sintomas depressivos. Importante ressaltar que a pesquisa não teve o objetivo de fazer 0 diagnóstico de depressão e sim determinar se existiam ou não a presença de sintomas depressivos e com que intensidade. Com o intuito de estudar a relação da frequência de profissionais da saúde que apresentavam sintomas de depressão e qual tipo de depressão representaria, foram utilizadas frequência e porcentagem, e estes dados podem ser observados na Tabela 3.

Tabela 3 - Frequência de profissionais da saúde que apresentaram sintomas depressivos e qual tipo de depressão representaria.

\begin{tabular}{ccc}
\hline Depressão & Frequência & Porcentagem \\
\hline Grave & 0 & 0 \\
Moderada & 2 & 0,20 \\
Leve & 3 & 0,30 \\
Não apresenta & 5 & 0,50 \\
\hline Total & 10 & 1
\end{tabular}

Fonte: Bonaldo PM e Portela CE, 2020.

Os resultados mostram que dos dez sujeitos de pesquisa, dois apresentaram depressão moderada e três apresentaram depressão leve. Os outros cinco participantes embora tivessem sintomas depressivos não foi suficiente para caracterizar uma depressão leve ou outro quadro mais grave. Novamente esses dados são alarmantes, visto que $50 \%$ dos profissionais participantes da nossa amostra do hospital apresentaram depressão leve ou moderada, este dado é bastante discrepante com os dados normativos onde apenas $24,1 \%$ da população, estaria enquadrada com depressão leve ou um caso mais grave (WENDT GW, et al., 2008).

O último instrumento foi o Questionário de Saúde Geral de Goldberg (QSG), apresentando as escalas de: Stress Psíquico, desejo de morte, desconfiança no próprio desempenho, distúrbios de sono, distúrbios psicossomáticos e saúde geral. A Tabela 4 traz as médias da população estudada em cada uma dessas escalas como pode ser visualizada abaixo: 
Tabela 4 - Média dos profissionais da saúde que dentro das escalas de Stress Psíquico, desejo de morte, desconfiança no próprio desempenho, distúrbios de sono, distúrbios psicossomáticos e saúde geral.

\begin{tabular}{lc}
\hline & Média \\
\hline Stress Psíquico & 2.43 \\
Desejo de Morte & 1.22 \\
Desconfiança Desempenho & 2.33 \\
Distúrbio Sono & 2.33 \\
Distúrbio Psicossomático & 2.37 \\
Saúde Geral & 2,12 \\
\hline
\end{tabular}

Fonte: Bonaldo PM e Portela CE, 2020.

A escala QSG vai de 1 a 4 pontos na escala Likert e apenas valores acima de 3 indicariam algum distúrbio e/ou comprometimento. Efetivamente podemos notar que nenhuma dessas médias estão acima de três o que poderia passar a falsa ilusão de que são índices "bons" para os trabalhadores. Esta parte dos dados será apresentada em percentil, são medidas que dividem a amostra ordenada (por ordem crescente dos dados) em 100 partes.

Então por exemplo um percentil de posição $90^{\circ}$ significa que determinado dado é maior que $90 \%$ da população mas que por outro lado inferior aos $10 \%$.

O que observa-se aqui e comparando com os estudos de normatização brasileira de Pasquali $L$, et al. (1996), começando pelo stress psíquico é que a média de 2,43 representa um percentil de mais de $75^{\circ}$ em relação a população geral, o desejo de morte de média de 1.22 representa um percentil acima de $55^{\circ}$ comparado com a norma, a desconfiança do desempenho de 2,33 está equiparado a um percentil acima de

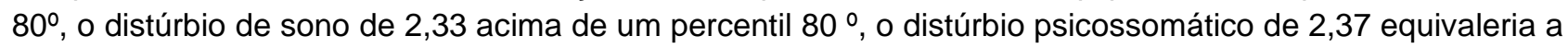
um percentil também acima de 80 e por último a saúde geral em um percentil acima de $75^{\circ}$. Todos esses índices demonstram como em média esses trabalhadores de saúde mental estão em um sofrimento qualitativamente e quantitativamente muito superior a população normal

Um dado bastante importante é se compararmos o nível de estresse dos trabalhadores do hospital pesquisado com um estudo realizado com outros profissionais de saúde mental. No caso citado, de acordo Santos AFO e Cardoso CL (2010), foi realizado com 25 trabalhadores de saúde mental de serviços ambulatoriais e de internação parcial de saúde mental de uma cidade do Interior do Estado de São Paulo.

Os serviços encontravam organizados segundo a Reforma Psiquiátrica e consistem em dispositivos comunitários de atenção em saúde mental caracterizados pelo modo de intervenção psicossocial.

O que se observou é que nessa população e, de acordo com este estudo, o qual usou o mesmo instrumento que esta pesquisa, o índice de estresse era de apenas $36 \%$ um índice muito inferior ao encontrado no que encontrou se no hospital pesquisado onde encontrou-se um índice de $70 \%$ de profissionais estressados na referida amostra.

\section{CONSIDERAÇÕES FINAIS}

O estudo problematiza as condições psíquicas dos trabalhadores de saúde mental. Além disto estudo também faz algumas sugestões para que esses profissionais expostos a estes riscos tenham também algum tipo de cuidado. Os pesquisadores sugerem alguns cuidados como: psicoterapia individual ou em grupo, atividades de relaxamento.

Podemos pensar também em estratégias coletivas como reunião de equipe para que este sofrimento seja problematizado, melhores condições ambientais e ergonômicas do trabalho e sobretudo que se possa ouvir esses trabalhadores para entender como eles querem ser ajudados. Fundamentalmente espera-se que uma instituição como o hospital pesquisado precisa de mais espaços coletivos, de convivência, de troca e que, de alguma forma, esse sofrimento possa ser falado ou simbolizado. 


\section{REFERÊNCIAS}

1. BRASIL. Lei no 8.080, de 19 de setembro de 1990. Lei Orgânica da Saúde. Dispõe sobre as condições para a promoção, proteção e recuperação da saúde, a organização e o funcionamento dos serviços correspondentes e dá outras providências. Brasília, set. 1990. Disponível em: http://www.planalto.gov.br/ccivil_03/leis//8080.htm. Acesso em: 02 junho. 2019

2. BECK AT, et al. An inventory for measuring depression. Archives of General Psychiatry, 1961. 4, 561-571.

3. CAMELO SH, ANGERAMI ELS. Sintomas de estresse nos trabalhadores atuantes em cinco núcleos de saúde da família. Rev. Latino-Am. Enfermagem, Ribeirão Preto, 2004. , v. 12, n. 1, p. 14-21.

4. DIAS S, et al. Síndrome de burnout e fatores associados em profissionais da área da saúde: um estudo comparativo entre Brasil e Portugal. Aletheia, Canoas. 2010 n. 32, p. 4-21.

5. ELY P, et al. Avaliação psicológica da depressão: levantamento de testes expressivos e autorrelato no Brasil. Aval. psicol. Itatiba. 2014 v. 13, n. 3, p. 419-426.

6. FERNANDES MA, MARZIALE MHP. Riscos ocupacionais e adoecimento de trabalhadores em saúde mental. Acta paul. enferm. São Paulo, 2014. v. 27, n. 6, p. 539-547.

7. LIPP MEN. Atualização Teórica sobre o Stress Emocional: o modelo quadrifásico. In: X Encontro Brasileiro de Psicoterapia e Medicina Comportamental, Campinas. Resumos, 2001. p. 100-101.

8. LIPP MEN. Manual do inventário de stress para adultos de Lipp. São Paulo: Casa do Psicólogo, 2005. p.76

9. LIPP MEN. O Modelo Quadrifásico do Stress. In: Lipp MEN (Org.). Mecanismo Neuropsicofisiológicos do Stress: teoria e aplicações clinicas. 1ed.SP: Casa do Psicólogo, 2003, v. 1, p. 17-21.

10. LIPP MEN, LIPP LMN. Proposal for a Four-Phase Stress Model. PSYCHOLOGY (IRVINE). 2019 v. 10, p. 14351443.

11. LIPP MEN. Pesquisa Stress brasil, site acessado: (2012) http://www.estresse.com.br/pesquisa/stress-brasil/ no dia 08/11/2017.

12. PASQUALI L, et al. Questionário de Saúde Geral de Goldberg (QSG): adaptação brasileira. Casa do Psicólogo. São Paulo, 1996.

13. PASQUALI L, et al. Questionário de Saúde Geral de Goldberg (QSG): adaptação brasileira e aplicação atualizada. Psicol Teor Pesqui 1994; 10:421-37.

14. ROSSETTI MO, et al. O inventário de sintomas de stress para adultos de lipp (ISSL) em servidores da polícia federal de São Paulo. Rev. Brasileira de terapias cognitivas. Rio de Janeiro , 2008. v. 4, n. 2,p. 108-120.

15. SANTOS AFO, CARDOSO CL. Profissionais de saúde mental: estresse e estressores ocupacionais stress e estressores ocupacionais em saúde mental. Psicol. estud., Maringá. 2010 v. 15, n. 2, p. 245-253.

16. SELYE H. The General Adaptation Syndrome. Annual Review of Medicine, 2, 1951 327-342.

17. SELYE H. Stress, a tensão da vida. São Paulo: Ibrasa - Instituição Brasileira de Difusão Cultural. 1959.

18. STRAUB RO. Psicologia da Saúde. Porto Alegre: ARTMED. 2003 p.122.

19. STRAUB RO. Introdução à Psicologia da saúde. Cap. 1. In: Psicologia aplicada a saúde. Porto Alegre: Artmed, 2005. p. 21-51.

20. WENDT GW, et al. Adaptação do Inventário de Depressão de Beck-II (BDI-II) para a População Brasileira. In: IX Salão de Iniciação Científica da PUCRS,2008, Porto Alegre. Anais do IX Salão de Iniciação Científica da PUC-RS... Porto Alegre: EDIPUCRS, p. 1-3. 